\title{
Neuropsychiatric Lupus in clinical practice
}

\section{Lúpus neuropsiquiátrico na prática clínica}

\author{
Helena Alessi', Livia Almeida Dutra', Pedro Braga-Neto ${ }^{2,3}$, José Luiz Pedroso', Fabio F. Toso', Cristiane
}

Kayser ${ }^{4}$, Orlando G. P. Barsottini ${ }^{1}$

\begin{abstract}
Systemic lupus erythematosus (SLE) is a chronic autoimmune disease involving multiple organs, characterized by the production of autoantibodies and the development of tissue injury. The etiology of SLE is partially known, involving multiple genetic and environmental factors. As many as $50 \%$ of patients with SLE have neurological involvement during the course of their disease. Neurological manifestations are associated with impaired quality of life, and high morbidity and mortality rates. Nineteen neuropsychiatric syndromes have been identified associated with SLE, and can be divided into central and peripheral manifestations. This article reviews major neuropsychiatric manifestations in patients with SLE and discusses their clinical features, radiological findings and treatment options.
\end{abstract}

Keywords: systemic lupus erythematosus; lupus vasculitis, central nervous system; seizures; myelitis; autoimmune diseases.

\section{RESUMO}

Lúpus eritematoso sistêmico (LES) é uma doença autoimune crônica que envolve múltiplos órgãos e sistemas, caracterizada pela produção de auto anticorpos e lesão tecidual. A etiologia do LES é parcialmente conhecida e envolve interação entre fatores genéticos e ambientais. Até $50 \%$ dos pacientes com LES apresentam envolvimento neurológico no decorrer da doença. Manifestações neurológicas estão associadas a prejuízo na qualidade de vida e altas taxas de mortalidade e morbidade. Foram identificadas 19 síndromes neuropsiquiátricas em pacientes com LES, divididas entre manifestações do sistema nervoso central e periférico. O objetivo deste artigo é revisar as manifestações neuropsiquiátricas mais importantes. Serão abordadas as características clínicas, os aspectos radiológicos e opções de tratamento dos eventos neuropsiquiátricos.

Palavras-chave: lúpus eritematoso sistêmico; vasculite associada ao lúpus, sistema nervoso central; convulsões; mielite; doenças autoimunes.

Systemic lupus erythematosus (SLE) is a chronic autoimmune disease involving multiple organs, characterized by the production of autoantibodies and the development of tissue injury ${ }^{1}$. The etiology of SLE is partially known and involves multiple genetic and environmental factors. Systemic lupus erythematosus more often affects nonWhite women of reproductive age and more severe forms are seen in people of African descent, Asians, Hispanics, and Indians ${ }^{1}$.

The pathogenesis of SLE is characterized by immune abnormalities including T-cell and B-cell hyperactivity, abnormal number and function of regulatory $\mathrm{T}$ cells, and immune complex deposition in various tissues. A characteristic feature of SLE is the production of autoantibodies against double-stranded DNA, histones and nucleosomes, and other chromatin components. In addition, immune complex and apoptotic cell removal is impaired ${ }^{1}$. These abnormalities could lead to the extracellular presence of chromatin triggering immune cells and activation of the innate and adaptive immune system ${ }^{2}$.

As many as $50 \%$ of patients with SLE have neurological involvement during the course of their disease ${ }^{3}$. Approximately $40 \%$ of neuropsychiatric SLE (NPSLE) cases are a consequence of the disease itself; other causes of NPSLE are infections, metabolic disorders, and side effects of drugs ${ }^{4}$. We review the major NPSLE manifestations and discuss their clinical features, radiological findings, and treatment options.

\footnotetext{
${ }^{1}$ Universidade Federal de São Paulo, Divisão de Neurologia Geral, Departamento de Neurologia e Neurocirurgia, São Paulo SP, Brasil;

¿Universidade Estadual do Ceará, Centro de Ciências da Saúde, Fortaleza CE, Brasil

${ }^{3}$ Universidade Federal do Ceará, Faculdade de Medicina, Departamento de Medicina Clínica, Divisão de Neurologia, Fortaleza CE, Brasil;

«Universidade Federal de São Paulo, Divisão de Reumatologia, São Paulo SP, Brasil.
}

Correspondence: Lívia Almeida Dutra; UNIFESP, Departamento de Neurologia; Rua Pedro de Toledo, 650; 04039-002 São Paulo SP, Brasil;

E-mail: iviaadutra@hotmail.com

Conflict of interest: There is no conflict of interest to declare.

Support: Coordenação de Aperfeiçoamento de Pessoal de Nivel Superior (CAPES) and grant \#2015/08833-7, São Paulo Research Foundation (FAPESP), supported this study.

Received 26 April 2016; Received in final form 09 August 2016; Accepted 11 August 2016. 


\section{NEUROPSYCHIATRIC SYSTEMIC LUPUS ERYTEMATOSUS}

The American College of Rheumatology identified 19 neuropsychiatric syndromes in SLE patients that can be divided into central and peripheral nervous system manifestations ${ }^{5}$ (Table 1). Although this classification includes syndromes with no clear physiopathological mechanism and is not specific for neuropsychiatric events caused exclusively by SLE, it helps the physician recognize any neurological involvement ${ }^{6}$.

NPSLE may be the first manifestation of the disease and its prevalence ranges from $21 \%$ to $95 \%^{5,6}$. Central nervous system (CNS) syndromes are more common than peripheral and may be further classified into diffuse or focal manifestations? Patients may present with single or multiple neuropsychiatric events, which may not relate to systemic disease activity ${ }^{5}$.

Risk factors associated with NPSLE include CNS damage or generalized SLE activity; previous neuropsychiatric events or other concurring neuropsychiatric manifestations; the presence of moderate to high titers of antiphospholipid antibodies (aPL) such as lupus anticoagulant and anticardiolipin, or anti- $\beta 2$ glycoprotein 1 (either IgG or IgM), especially in cerebrovascular disease, myelopathy, cognitive dysfunction, seizures and movement disorders; and anti-ribosomal P protein antibodies (anti-P antibodies), that have been associated with lupus psychosis in some studies ${ }^{6,7,8,9}$.

Table 1. Nineteen case definition for NPSLE syndromes.

\begin{tabular}{l}
\hline Central nervous system \\
\hline Headache \\
Seizure disorders \\
Cerebrovascular disease \\
Demyelinating syndrome \\
\hline Myelopathy \\
\hline Movement disorder \\
\hline Aseptic meningitis \\
\hline Cognitive dysfunction \\
\hline Mood disorder \\
Anxiety disorder \\
Psychosis \\
Acute confusional state \\
\hline Peripheral nervous system \\
\hline Mononeuropathy \\
\hline Polyneuropathy \\
\hline Cranial neuropathy \\
\hline Acute inflammatory demyelinating polyradiculoneuropathy \\
(Guillain-Barré syndrome) \\
\hline Plexopathy \\
\hline Autonomic disorder \\
\hline Myasthenia gravis \\
\hline
\end{tabular}

NPSLE: Neuropsychiatric systemic lupus erythematosus.
Neurological manifestations are associated with impaired quality of life, and high morbidity and mortality rates. Notably, involvement of the lungs, kidneys and CNS can cause serious sequelae ${ }^{6}$. Since there are no biomarkers of CNS activity, the diagnosis of NPSLE is often made by ruling out secondary NPSLE. Potential pathogenic mechanisms in primary NPSLE are direct action of intrathecal inflammatory cytokines, blood-brain barrier (BBB) disruption, accelerated atherosclerosis and thrombotic vasculopathy caused by aPL antibodies ${ }^{4}$. Autoantibodies may also bind to neurons leading to neuronal dysfunction and apoptosis ${ }^{7,10}$ (Table 2).

\section{Headache}

Headache is a common, non-specific symptom for SLE or other rheumatic diseases. Although tension-type headache is the most frequent primary headache syndrome in the general population, there are reports of higher prevalence of migraine in SLE when compared to controls ${ }^{11,12}$. Nonetheless, a recent meta-analysis did not confirm this finding ${ }^{11}$. Headache in SLE is not associated with a higher frequency of magnetic resonance imaging (MRI) lesions, disease activity, and biomarkers such as aPL, anti-P, and glutamate receptor antibodies (anti-NR2) $)^{12,13}$.

Focal neurological signs, fever or concomitant infection, presence of antiphospholipid syndrome (APS), immunosuppression, use of anticoagulants, altered mental status, meningismus and generalized SLE activity are indicative of secondary causes of headache and require further investigation with brain imaging and cerebrospinal (CSF) analysis ${ }^{8,14}$. It is important to rule out meningitis, cerebral venous sinus thrombosis (mostly in patients with aPL) and cerebral or subarachnoid hemorrhage ${ }^{8}$. In addition, secondary headaches may be caused by posterior reversible encephalopathy syndrome ${ }^{14}$.

Non-steroidal anti-inflammatory drugs and triptans can be used for pain relief. Migraine prevention therapy are $\beta$-blockers, anticonvulsants such as valproic acid and topiramate, and antidepressants ${ }^{15}$.

Table 2. Know autoantibodies association with NPSLE.

\begin{tabular}{|c|c|c|}
\hline Autoantibodies & Syndromes & Source \\
\hline LA & CVD, CD, seizures & Sciascia, 2014 \\
\hline $\mathrm{aCL}$ & $\begin{array}{l}\text { CVD, CD, seizures } \\
\text { and eplepsy }\end{array}$ & Sciascia, 2014 \\
\hline$a \beta G P I$ & $\begin{array}{l}\text { Angina and arterial or } \\
\text { venous thrombosis }\end{array}$ & Sciascia, 2014 \\
\hline Anti-Sm & ACS & Hirohata, 2014 \\
\hline Anti-P & Psycosis & $\begin{array}{c}\text { Bonfa, 1987; } \\
\text { Sciascia, } 2014\end{array}$ \\
\hline Anti-NMDA & $C D$ & Faust, 2010 \\
\hline Anti-NR2 & $C D$ & Lauvsnes, 2014 \\
\hline AQP4 & NMO & Bertsias, 2010 \\
\hline
\end{tabular}

NPSLE:Neuropsychiatric systemic lupus erythematosus;LA:Lupus anticoagulant; CVD: Cerebrovascular disease; CD: Cognitive dysfunction; aCL: anticardiolipin; ACS: Acute confusional state;AQP4:Aquaporin 4; NMO: Neuromyelitis optica. 


\section{Cognitive dysfunction}

Cognitive dysfunction (CD) is defined as a cognitive decline from a previous level of mental functioning documented by neuropsychological assessments adapted to the target population ${ }^{5,16}$. The main cognitive domains found to be compromised in SLE are attention, processing speed and memory ${ }^{5,16}$. The American College of Rheumatology recommends a one-hour neuropsychological battery for CD evaluation in SLE patients. It assesses simple and complex attention, verbal and visual memory, visual spatial processing, language (verbal fluency), reasoning/problem solving, psychomotor speed and executive function (Table 3).

Cognitive decline is defined by scores that fall between 1.5 and 1.9 standard deviations below the mean in one or more cognitive domains. Cognitive impairment is defined by scores that fall 2.0 standard deviations below the mean ${ }^{16}$. Cognitive dysfunction is defined as focal if impairment exists on measures within one domain, or multifocal if impairment exists on

Table 3. Proposed neuropsychological battery for SLE.

\begin{tabular}{lc}
\hline Tests & Domains \\
\hline North American Adult Reading Test & Premorbid IQ \\
\hline Digit Symbol Test & Processing Speed \\
\hline Trail Making Test & Attention \\
Stroop Color Test & Complex Attention \\
California Verbal Learning Test & Verbal Memory \\
Rey Complex Figure & Visual Memory \\
Letter Number Sequencing & Working Memory \\
Controlled Oral Word Association Test & Phonemic Verbal Fluency \\
Animal Naming & Semantic Verbal Fluency \\
\hline Finger Tapping & Motor Speed \\
\hline
\end{tabular}

SLE: systemic lupus erythematosus. measures spanning two or more domains ${ }^{16}$. Screening tests, i.e. Mini Mental State Examination, are non-specific and have low diagnostic sensitivity for $\mathrm{CD}^{5}$. Despite that, the Montreal Cognitive Assessment is an appropriate screening tool for CD because it features a frontal subcortical profile of cognitive impairment in SLE. In American patients with SLE, the Montreal Cognitive Assessment tool had a sensitivity of $83 \%$ and specificity of $73 \%$ for $\mathrm{CD}$ using a cutoff score of $26^{17}$.

Cognitive dysfunction in SLE is associated with aPL antibodies, steroid use, diabetes and low level of education. Although depressive symptoms may reduce cognitive function, $\mathrm{CD}$ is not fully explained by depression ${ }^{18}$. In a comparison of neuropsychological scores between patients with depression and those with depression and SLE, the latter showed even lower scores ${ }^{18}$.

Cerebral atrophy, white matter lesions and cerebral infarction have been correlated with the severity of $\mathrm{CD}^{8}$ (Figure 1). An apparently normal MRI in CD may be associated with microstructural and metabolic changes in white matter tissue suggesting potentially immune-mediated myelinopathy, which is best seen by spectroscopy and other advanced MRI techniques ${ }^{19}$. The pathophysiology of CD remains to be fully understood and may involve vascular abnormalities, intrathecal inflammatory cytokines and BBB disruption. Autoantibodies might also contribute to the pathogenesis of CD in SLE7 . Systemic lupus erythematosus patients with lupus anticoagulant are three times more likely to have impaired neuropsychological functioning, with working memory dysfunction, probably due to white matter changes and microvascular thrombosis ${ }^{20}$. Murine models have shown that anti-NMDA antibodies elicit neuronal death producing $\mathrm{CD}$ and emotional disturbance ${ }^{21}$. A recent study has shown higher levels of anti-NR2 antibodies in the CSF and reduced hippocampal gray matter in SLE patients when compared with controls ${ }^{22}$.
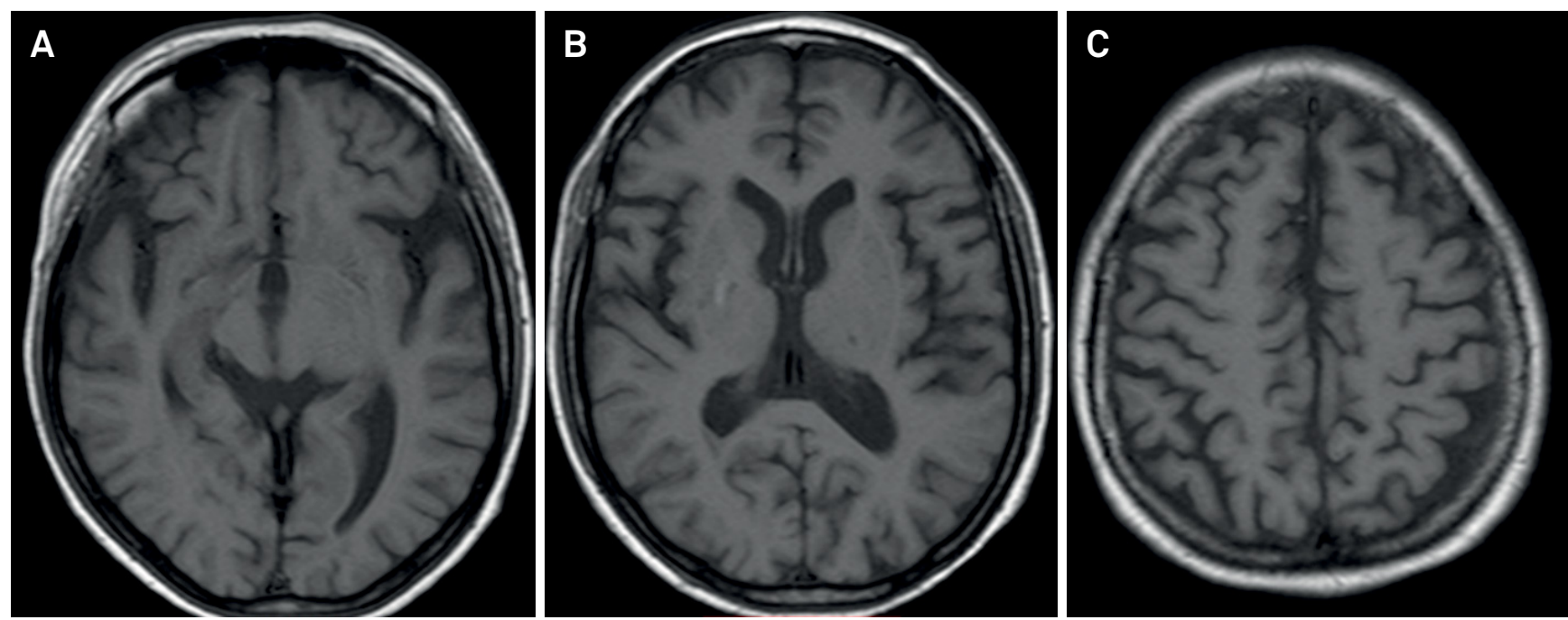

Figure 1. Cortical atrophy in SLE. A 32-year-old female with SLE had a prior stroke due to SLE vasculitis. After four years, she developed mononeuritis multiplex and cognitive impairment. She was negative for aPL antibodies. (A) Brain MRI T1 sequence. Observe the Sylvian fissure. (B, C) Global cortical atrophy. 
The treatment for $\mathrm{CD}$ remains uncertain. Identification and management of secondary or exacerbating causes of $\mathrm{CD}$ is recommended ${ }^{19}$. Patients may respond to methylphenidate or prednisone $(0.5 \mathrm{mg} / \mathrm{kg})^{19}$. Aspirin is only indicated for patients with cardiovascular comorbidities to improve cognitive performance; and memantine, an NMDA-receptor antagonist, prevents cognitive impairment from anti-NR2 antibodies in the murine model, but it has not been effective in SLE patients ${ }^{23}$. Cognitive rehabilitation and psychoeducation are complementary treatments that can help improve subjective and objective cognitive complaints ${ }^{8}$.

\section{Seizures}

Epilepsy occurs in $12 \%$ to $22 \%$ of patients with SLE and is associated with increased morbidity and mortality ${ }^{8}$. A single isolated seizure is more frequently seen and a generalized tonic-clonic seizure is the most common type (67\% to $88 \%$ ), but a simple partial and complex seizure can also occur ${ }^{8}$. Abnormalities on the EEG are common (60\% to 70\%) in SLE, and epileptiform EEG patterns suggest the seizure is likely to recur (73\% positive predictive value, $79 \%$ negative predictive value $)^{24}$. Patients with seizures show more gray matter hyperintensities on MRI images and may develop brain atrophy ${ }^{13}$.

Inflammatory processes are believed to play a major role in the pathogenesis of epileptic seizures ${ }^{25}$. Ischemic vascular disease and antibodies that bind to cerebral tissues such as anticardiolipin and anti-Sm have been associated with seizures $^{24}$. Epilepsy in SLE has also been associated with APS, disease activity, multiple NPSLE manifestations (i.e., psychosis, stroke), and severe baseline organ damage ${ }^{24,26}$. Although seizure is classified as a diffuse CNS manifestation, it may develop in focal and inflammatory NPSLE. For that reason, the evaluation of patients with seizures should include brain imaging and CSF analysis to rule out infections, vasculitis, and mechanisms suggestive of focal NPSLE.

Posterior reversible encephalopathy syndrome is a clinical-radiological syndrome characterized by seizures, altered mental status, and visual impairment ${ }^{27}$. It is an uncommon condition with a good prognosis, usually reversible and rarely recurrent ${ }^{14}$. An MRI in this syndrome shows bilateral asymmetrical isointensities or hypointensities in T1, hyperintensities in T2 and fluid-attenuated inversion recovery sequences in the parietal-temporal-occipital regions ${ }^{27}$. Approximately $50 \%$ of patients require treatment with both anticonvulsants and antihypertensive drugs ${ }^{27}$.

Long-term anticonvulsants are recommended for patients with recurrent seizures or risk of recurrence (i.e., recurrent seizures in 24 hours, EEG abnormalities, prior brain injury with structural abnormalities in brain MRI) ${ }^{8}$. Immunosuppressive therapy is indicated for those with systemic disease activity.

\section{Acute confusional state}

Acute confusional state is a disturbance in consciousness or alertness, and subsequent attentional deficits that are accompanied by cognitive decline and/or affect or mood changes. It has been associated with the presence of anti-NR2 antibodies and anti-Sm antibodies in the $\mathrm{CSF}^{10,28}$. The pathogenic mechanism behind this diffuse neuropsychiatric manifestation seems to be primarily inflammatory with increased production of inflammatory mediators, BBB disruption, and intrathecal immune complex formation ${ }^{6}$. Risperidone ( $2 \mathrm{mg} /$ day) may be a therapeutic option for an acute confusional state ${ }^{29}$. Yet, corticosteroids and immunosuppression should be considered depending on the patient's condition ${ }^{8}$.

\section{Psychosis, depression and anxiety}

A systematic review found a prevalence of $17 \%$ to $75 \%$ of depressive disorders among patients with SLE ${ }^{30}$. Mood disorders were associated with disease activity, high prednisone doses ( $\geq 20 \mathrm{mg}$ ), cutaneous disease, and longitudinal extensive transverse myelitis ${ }^{31}$. Adverse drug events may also contribute to the occurrence or exacerbation of depressive symptoms in some patients ${ }^{32}$. There is no evidence on the use of neuroimaging or serological markers for diagnosing mood and anxiety disorders, although anti-P and anti-NMDA receptor autoantibodies have been associated with a higher incidence of depression in patients with $\mathrm{SLE}^{8,32}$. Serum levels of tumor necrosis factor alpha are also increased in SLE patients with mood and anxiety disorders suggesting the role of inflammation in depression ${ }^{31}$.

Anti-P antibodies have been specifically associated with psychosis in patients with SLE, although some studies have failed to verify this association ${ }^{7,9}$. High levels of anti-P antibodies found in serum samples of SLE patients with psychosis were compared to those of SLE patients with other neuropsychiatric manifestations, patients with non-SLE psychosis, and controls. Anti-P antibody levels were 5- to 30-fold higher during the active phase of SLE psychosis, but not during other SLE manifestations ${ }^{9}$. In fact, several in vivo and in vitro studies have shown that anti-P antibodies can bind to neuronal antigens, penetrate neuronal cells, and inhibit protein synthesis in neuronal cells including in hippocampal neurons. Moreover, a recent study has shown that anti-P antibodies interact with neuronal antigens leading to neuronal apoptosis ${ }^{32}$.

Both psychosis and major depressive disorders due to SLE are rare, while steroid-induced psychosis is an uncommon side effect. Glucocorticoids and immunosuppressive therapy may be considered for psychosis associated with SLE, especially in the presence of generalized disease activity ${ }^{8}$.

\section{Cerebrovascular disease}

Cerebrovascular events account for $10 \%$ to $15 \%$ of deaths in SLE patients ${ }^{33}$. Ischemic stroke (IS) is the most common condition. Patients with SLE have a twofold increase in the risk of IS, a threefold increase in the risk of intracerebral stroke, and a fourfold increase in the risk of subarachnoid hemorrhage compared to the general population $^{33}$. Intracerebral and subarachnoid hemorrhages are 
infrequent in SLE patients, and affect mostly young individuals. They usually occur in the first year following the diagnosis, and result in high mortality and long-term morbidity ${ }^{33}$.

Systemic lupus erythematosus disease severity, hypertension and hyperlipidemia were independent predictors of stroke and stroke severity in a follow-up study ${ }^{34}$. However, traditional risk factors do not fully explain the high prevalence of IS in patients with SLE. Accelerated atherosclerosis, and inflammatory mediators such as complement components, cytokines and aPL antibodies may also play a role in the development of cerebrovascular disease 4 . It is noteworthy that high cumulative doses of steroids could also be a risk factor for accelerated atherosclerosis, leading to increased risk of stroke ${ }^{33}$. Vasculitis is a rare cause of stroke in SLE, accounting for $7 \%$ of cases in some case series.

Patients with SLE and stroke should be routinely screened for APS, which is characterized by the presence of aPL antibodies, (arterial or venous) vascular thrombosis and pregnancy complications including eclampsia or pre-eclampsia and miscarriage ${ }^{35}$ (Figure 2). Stroke in APS is either thrombotic or embolic ${ }^{35}$. Patients with SLE and APS may also present with renal involvement and valvulopathy due to immune complex deposition. One-third or more of APS strokes are due to embolism associated with cardiac valve vegetations, and some patients may have sterile endocarditis ${ }^{35}$.

A recent systematic review pointed to a fivefold increase in the risk of IS or transient ischemic attack in patients with aPL antibodies compared to controls ${ }^{36}$. The RATIO (Risk of Arterial Thrombosis in Relation to Oral Contraceptives) study showed that lupus anticoagulant is a major risk factor for IS, particularly in young women. Anti- $\beta 2$-glycoprotein is also associated with higher IS risk ${ }^{37}$. Lupus anticoagulant is a stronger predictor of thrombosis compared to anticardiolipin and usually involves large and small vessels.

Modifiable risk factors should be focused upon as part of primary and secondary IS prevention strategies for patients with $\mathrm{SLE}^{38}$. Long-term oral anticoagulant is recommended for patients with APS and SLE, to prevent recurrence of both arterial and venous thrombosis, and also for patients with a high-risk cardioembolic source. Glucocorticoids and immunosuppressive therapies do not reduce the risk of $\mathrm{IS}^{38}$.

\section{Movement disorders}

Movement disorders are uncommon neuropsychiatric manifestations in SLE. Nonetheless, parkinsonism, myoclonus and dystonia were described and an autoimmune or thromboembolic disease mechanism has been proposed for their occurrence ${ }^{39}$. Chorea is the most described manifestation and may be the first manifestation in SLE occurring in $2 \%$ of adult patients, predominantly in women. It can be unilateral or bilateral and remit spontaneously. Current evidence suggests an autoimmune mechanism related to aPL antibodies, but focal cerebral ischemia is rarely evidenced ${ }^{39}$.

Sydenham chorea is the most common cause of acute chorea in children and it is one important differential diagnosis in chorea associated with lupus, especially in pediatric patients. Sydenham chorea is an autoimmune disorder and most cases develop following a rheumatic fever or streptococcal infection ${ }^{40}$. Sydenham chorea and lupus may have similar findings in brain single-photon emission computed tomography. Both diseases showed hyperconcentration of the radiotracer in the basal ganglia, possibly due to changes in the basal ganglia microcirculation or to a BBB abnormality secondary to the inflammatory process ${ }^{41}$.
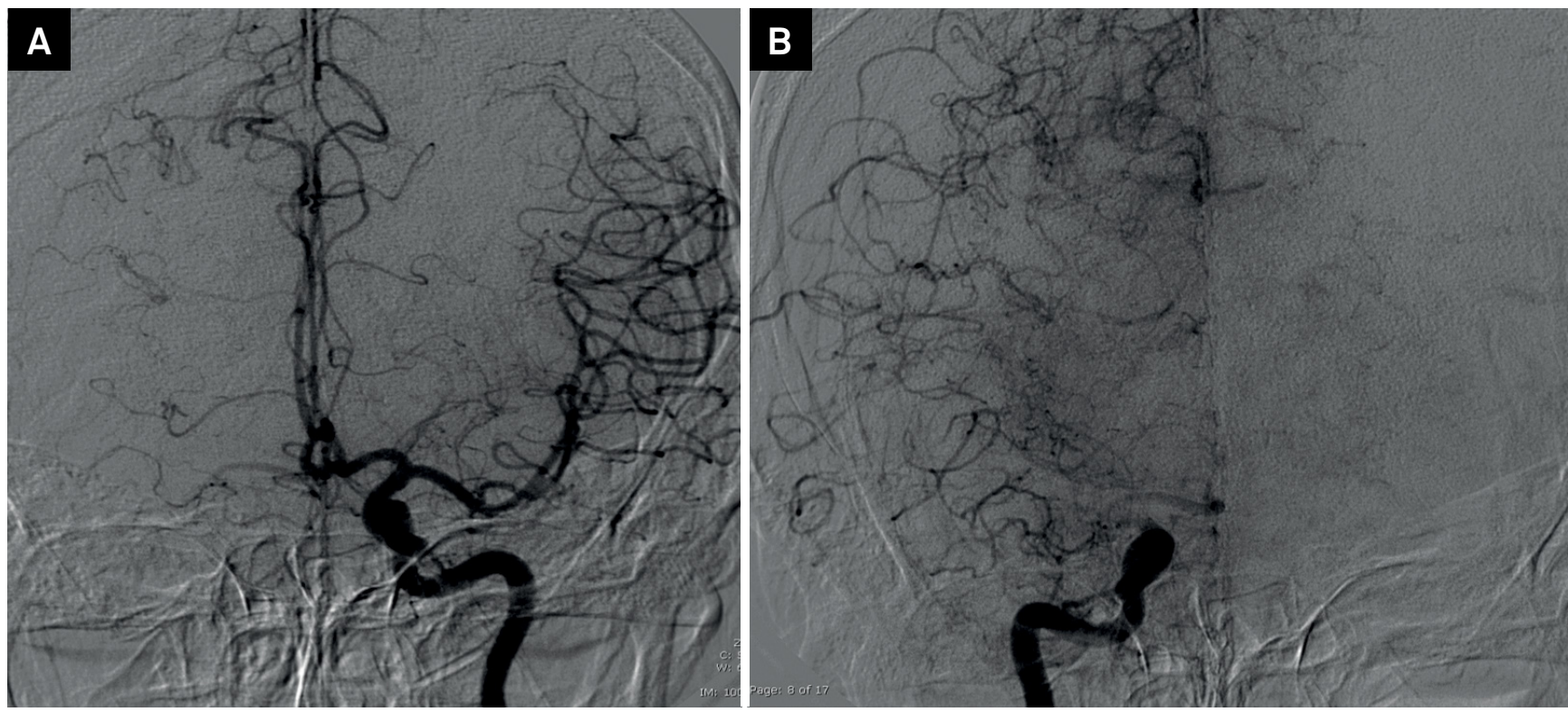

Figure 2. Large vessel thrombosis in antiphospholipid syndrome. (A) Digital arteriography showing normal left carotid artery. (B) Right carotid thrombosis in an SLE patient with stroke and positive lupus anticoagulant antibodies. 
Parkinsonism may occur in children or adult patients. Dopaminergic drugs as well as immunomodulatory therapy may improve their symptoms. Antiphospholipid syndrome-related parkinsonism is associated with vasculopathy, poor response to levodopa and cerebral infarction. Systemic lupus erythematosus patients with APS may also present with tremor, tics, myoclonus and corticobasal degeneration-like syndrome ${ }^{42}$.

Focal dystonia including torticollis and blepharospasm have also been reported. They may respond to immunomodulatory therapies. Some patients with SLE may have complex movement disorders including an association of hypokinetic and hyperkinetic manifestations that are frequently associated with other neuropsychiatric symptoms ${ }^{42}$.

\section{Aseptic meningitis}

The incidence of aseptic meningitis in SLE patients has been estimated at $1.4 \%$ to $1.6 \%$ in retrospective studies. It can occur at any time during the disease course, usually during flares ${ }^{32,43}$. Because patients are often receiving immunosuppressant agents, bacterial, viral, fungal and tuberculosis infection must be ruled out ${ }^{43}$. Clinical features of aseptic meningitis include headache and altered mental status. Cerebrospinal fluid analysis may reveal lymphocytic cells and protein. Although spontaneous remission may occur, most cases are effectively treated with glucocorticoids and immunosuppressive therapy ${ }^{8}$.

\section{Myelopathy}

Lupus myelitis occurs in $1 \%$ to $1.5 \%$ of $\operatorname{cases}^{44}$. It manifests as transverse myelitis or asymmetrical spinal cord syndrome with hemiparesis, tetraparesis, bladder involvement, neuropathic pain, and presence of a sensory level. Acute transverse myelitis is the first clinical manifestation in nearly half of the patients with SLE and it usually occurs within the first five years after diagnosis ${ }^{44}$. Underlying pathophysiological mechanisms are not yet known. Histopathology findings have revealed ischemic/thrombotic myelopathy (especially in acute cases) or localized acute inflammation ${ }^{44}$.

Systemic lupus erythematosus myelitis has two distinct clinical patterns: gray-matter and white-matter myelitis. Patients with gray-matter myelitis often have a prodromal phase of fever and urinary retention at onset that rapidly evolves to paraplegia during disease activity. An MRI reveals spinal cord swelling and enhancement ${ }^{45}$. White-matter myelitis has been associated with a less severe presentation and slower progression, characterized by upper motor neuron spasticity and hyperreflexia ${ }^{45}$.

The CSF analysis may show mild pleiocytosis exceeding 10 cells $/ \mathrm{mm}^{3}$, but polymorphonuclear predominance has also been reported. There is often an elevation of IgG in $\mathrm{CSF}^{44}$. An MRI may show increased signal and longitudinal extensive transverse myelitis involving at least three vertebral segments has been observed in $91.7 \%$ of gray-matter myelitis ${ }^{45}$. There may be no MRI abnormalities in some cases. Approximately $30 \%$ of patients may have brain involvement, and therefore it is advisable to perform brain $\mathrm{MRI}^{44}$.

There may also be a co-occurence of SLE with neuromyelitis optica (NMO). So patients should be tested for NMO-IgG anti-aquaporin 4 (AQP4) antibodies ${ }^{8}$. Neuromyelitis opticaIgG is positive in $10 \%$ of SLE cases with myelitis and approximately $20 \%$ of patients may fulfill clinical criteria for NMO as reported in some series. Because myelitis in SLE may respond to cyclophosphamide whereas patients with NMO tend to have a worse outcome, it is important to test patients at the first manifestation of the disease before immunosuppression and to repeat it during the disease course (Figure 3).

In the presence of myelitis, overlap of APS, Sjögren's syndrome, and NMO should be investigated ${ }^{32}$. The prevalence of aPL ranges from $18 \%$ to $60 \%$ in SLE patients with myelitis. Spinal cord necrosis secondary to thrombosis has been proposed as an etiological factor for transverse myelitis in SLE ${ }^{32}$.

Treatment involves therapy with high-dose steroids followed by intravenous cyclophosphamide. The addition of plasmapheresis to a regimen of intravenous cyclophosphamide and steroids led to higher remission rates ${ }^{32}$. Relapse of lupus-associated acute transverse myelitis is common (50-60\%) during steroid tapering. Severity of neurological impairment (muscle strength $<3 / 5$ ) is the most important prognostic variable ${ }^{46}$. Other prognostic variables in SLE-associated myelitis are urinary catheter use at the neurological nadir and no cyclophosphamide therapy. Only a small proportion of patients fully recover.

\section{Peripheral nervous system involvement}

Some SLE patients may present with variable peripheral nervous system involvement $(2-3 \%)^{5}$ (Table 1). Clinical symptoms include combined paresthesia, pain, autonomic dysfunction, peripheral ataxia, weakness, and atrophy. Peripheral neuropathy is most often seen in older patients at diagnosis, and those with CNS manifestations and active systemic disease. Differences in disease activity may account for variation in the frequency and spectrum of peripheral neuropathies in different cohorts ${ }^{47,48}$. Electroneuromyography can help identify neurophysiological patterns. Analysis of the CSF is useful in inflammatory demyelinating polyradiculoneuropathy. Nerve biopsy is rarely useful in such patients ${ }^{8}$.

Mild distal symmetric axonal sensory or sensorimotor neuropathy is the most common subtype of peripheral involvement, whereas mononeuropathies are the second most common presentation. Patients may present with sudden weakness requiring aggressive immunosuppressive therapy. Although small-fiber neuropathy is not included in the American College of Rheumatology definitions, Oomatia reported a prevalence of $17.1 \%$ in SLE patients with peripheral involvement ${ }^{5,49}$. Electrodiagnostic studies are usually normal and a skin biopsy demonstrates loss of intraepidermal nerve fibers. There are two distinct clinicopathologic 

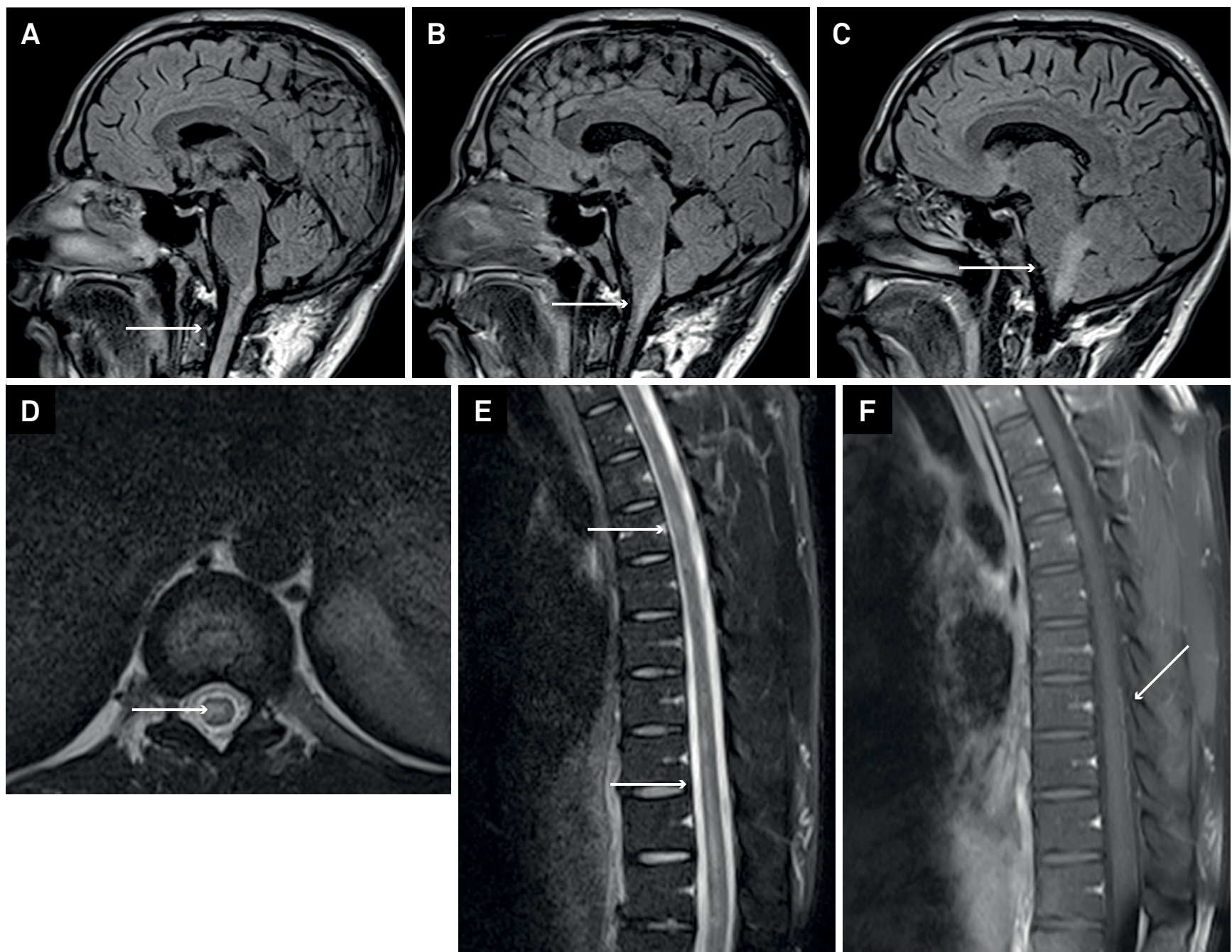

Figure 3. Myelitis in SLE. (A, B, C) Brain MRI fluid-attenuated inversion recovery sequence. Arrows: extensive myelitis extending to the area postrema in the dorsal medulla. (D) Axial T2 sequence showing spinal cord involvement in the dorsal region. (E) Sagittal T2 sequence. Arrows: Observe the multiple lesions, one involving more than three spinal segments. (F) Sagittal T1 sequence: pial contrast enhancement. Patient with SLE and APS negative for AQP4, had an isolate demyelinating episode, was treated with plasmapheresis, cyclophosphamide and steroids with complete recovery.

entities: length-dependent, small-fiber neuropathy with a stocking-and-glove distribution and abnormal skin biopsy findings restricted to the distal leg and non-length-dependent, small-fiber neuropathy with an unorthodox pattern of patchy, asymmetric, and proximal neuropathic pain that can affect the face, torso, and proximal extremities ${ }^{50}$.

Mononeuritis multiplex is found in $33 \%$ of SLE patients with peripheral involvement, usually with sudden weakness in different nerve territories ${ }^{51,52}$. It can occur at any time during disease course, either at the onset of SLE or later during its evolution ${ }^{51,52}$. In most cases, the onset was dramatic with sudden weakness in different nerve territories. These patients, in general, had substantially higher disease activity, showed more severe deficits and often required more aggressive immunosuppressive therapy.

Chronic inflammatory demyelinating polyneuropathy and acute inflammatory demyelinating polyneuropathy are severe. Forms of chronic inflammatory demyelinating polyneuropathy can present before or at the onset of another clinical manifestations of SLE ${ }^{53}$. Acute inflammatory demyelinating polyneuropathy, an ascending motor radiculoneuropathy that resembles Guillain-Barré syndrome clinically and electrodiagnostically, is a relatively rare condition, affecting up to $1 \%$ of the lupus patients ${ }^{54}$.

Symptomatic therapy alone may be considered for mild peripheral neuropathy, especially when there is no motor impairment ${ }^{32}$. Glucocorticoids alone or together with immunosuppressive therapy have been used with good outcomes (60-75\% response rate). Intravenous immunoglobulin, plasma exchange, and rituximab have been used in severe and refractory cases ${ }^{8}$.

\section{Cranial neuropathies}

Cranial neuropathies may involve the eighth nerve, the oculomotor nerve (third, fourth and sixth), and less commonly the fifth and the seventh nerves ${ }^{8}$. Cranial neuropathies 
have been reported in 5-42\%, rarely as an isolated manifestation $^{55}$. When the oculomotor, trochlear and abducent nerves are involved, myasthenia gravis should be ruled out. Different mechanisms have been proposed such as vasculitis secondary to SLE, microinfarction of the capillaries or arterioles in the temporal bone, and thrombosis in the otologic region ${ }^{56}$. Autoimmune sensory neural hearing loss in SLE is associated with a poor prognosis.

Optic neuropathy is infrequent $(<1 \%)$ and can manifest as optic neuritis and ischemic/thrombotic optic neuropathy, which is usually unilateral and related to aPL ${ }^{57}$. Only $50 \%$ of patients with SLE-associated optic neuritis recovered complete visual acuity (visual acuity better than $20 / 25)^{58}$. Bilateral or chiasm involvement, severe pain accentuated by ocular movements and profound visual impairment are characteristic of SLE-associated optic neuritis. The differential diagnosis includes NMO and multiple sclerosis.

More recently, studies with small samples reported a frequency of audiovestibular symptoms between $25 \%$ and $67 \%$ in SLE patients ${ }^{59,60}$. Such manifestations include sensorineural hearing loss, often accompanied by vertigo and tinnitus and other symptoms compatible with Menière's disease ${ }^{60}$. Autoimmune sensory neural hearing loss in SLE is associated with a poor prognosis.

Patients with SLE may present with asymptomatic cranial neuropathies, and the eighth nerve is the most commonly affected, followed by the seventh, the second, and the fifth nerves. Anti-ribosomal P protein and anti-DNA antibodies are associated with such subclinical manifestation ${ }^{55}$.

In conclusion, NSLE exhibits variable clinical manifestations and a heterogeneous disease course and prognosis. Since there are no biomarkers for CNS involvement and disease activity, diagnosis should be based on clinical features as well as imaging and laboratory findings. Central nervous system infections, metabolic abnormalities, valvular heart disease, and adverse drug effects should be ruled out. All patients with NPSLE should be routinely screened for APS, since overlap may occur even in non-ischemic manifestation.

For therapeutic purposes, the most important decision is to classify primary NPSLE into either ischemic or inflammatory phenotypes and treatment should be individualized ${ }^{61}$. Severe inflammatory phenotypes should receive immunosuppressant and/or steroid therapy, whereas ischemic phenotypes should receive aspirin or anticoagulation. In mild cases, therapy consists of antidepressants, anticonvulsants, or antipsychotics only.

\section{Acknowledgments}

We thank Dr. Alexandre Wagner Silva de Souza and Prof. Emilia Inoue Sato for their invaluable support.

\section{References}

1. Hahn B. Lúpus eritematoso sistêmico. In: Fauci AS, Braunwald E, Kasper DL et al., editors. Harrison medicina interna. 17a ed. Rio de Janeiro: McGraw-Hill Interamericana do Brasil; 2008. p. 2075-83.

2. Rother N, Vlag J. Disturbed T cell signaling and altered th17 and regulatory $T$ cell subsets in the pathogenesis of systemic lupus erythematosus. Front Immunol. 2015;6:610. doi:10.3389/fimmu.2015.00610

3. Kozora E, Erkan D, West SG, Filley CM, Zhang L, Ramon G et al. Site differences in mild cognitive dysfunction (MCD) among patients with systemic lupus erythematosus (SLE). Lupus. 2013;22(1):73-80. doi:10.1177/0961203312468963

4. Pamfil C, Fanouriakis A, Damian L, Rinzis M, Sidiropoulos P, Tsivgoulis G et al. EULAR recommendations for neuropsychiatric systemic lupus erythematosus vs usual care: results from two European centres. Rheumatology (Oxford). 2015;54(7):1270-8. doi:10.1093/rheumatology/keu482

5. Liang M, Corzillius M, Bae S, Lew RA, Fortin PR, Gordon C. The American College of Rheumatology nomenclature and case definitions for neuropsychiatric lupus syndromes. Arthritis Rheum. 1999;42(4):599-608. doi:10.1002/1529-0131 (199904)42:4<599::AID-ANR2>3.0.CO;2-

6. Hanly JG. Diagnosis and management of neuropsychiatric SLE. Nat Rev Rheumatol. 2014;10(6):338-347. doi:10.1038/nrrheum.2014.15

7. Sciascia S, Bertolaccini ML, Roccatello D, Khamashta MA, Sanna G. Autoantibodies involved in neuropsychiatric manifestations associated with systemic lupus erythematosus: a systematic review. J Neurol. 2014;261(9):1706-14. doi:10.1007/s00415-014-7406-8
8. Bertsias G, loannidis J, Aringer M, Bollen E, Bombardieri S, Bruce IN et al. EULAR recommendations for the management of systemic lupus erythematosus with neuropsychiatric manifestations: report of a task force of the EULAR standing committee for clinical affairs. Ann Rheum Dis. 2010;69(12):2074-82. doi:10.1136/ard.2010.130476

9. Bonfa E, Golombek SJ, Kaufman LD, Skelly S, Weissbach H, Brot $\mathrm{N}$ et al. Association between lupus psychosis and antiribosomal P protein antibodies. N Engl J Med. 1987;317(5):265-71. doi:10.1056/NEJM198707303170503

10. Hirohata S, Sakuma Y, Yanagida T, Yoshio T. Association of cerebrospinal fluid anti-Sm antibodies with acute confusional state in systemic lupus erythematosus. Arthritis Res Ther. 2014;16(5):450. doi:10.1186/s13075-014-0450-z

11. Mitsikostas DD, Sfikakis PP, Goadsby PJ. A meta-analysis for headache in systemic lupus erythematosus: the evidence and the myth. Brain. 2004;127(5):1200-9. doi:10.1093/brain/awh146

12. Hanly J, Urowitz MB, O'Keeffe AG, Gordon C, Bae SC, Sanchez-Guerrero J et al. Headache in systemic lupus erythematosus: Results from a prospective, international inception cohort study. Arthritis Rheum. 2013;65(11):2887-97. doi:10.1002/art.38106

13. Jeong HW, Her M, Bae JS, Kim SK, Lee SW, Kim HK et al. Brain MRI in neuropsychiatric lupus: associations with the 1999 ACR case definitions. Rheumatol Int. 2015;35(5):861-9. doi:10.1007/s00296-014-3150-8

14. Schwartz N, Mitnick HJ, Nowatzky J. Headaches related to rheumatologic disease. Curr Pain Headache Rep. 2013;17(12). doi:10.1007/s11916-013-0381-4 
15. Diener HC, Charles A, Goadsby PJ, Holle D. New therapeutic approaches for the prevention and treatment of migraine. Lancet Neurol. 2015;14(10):1010-2. doi:10.1016/S1474-4422(15)00198-2

16. Mikdashi J, Esdaile J, Alarcón GS, Crofford L, Fessler BJ, Shanberg $L$ et al. Proposed response criteria for neurocognitive impairment in systemic lupus erythematosus clinical trials. Lupus. 2007;16(6):418-25. doi:10.1177/0961203307079044

17. Adhikari T, Piatti A, Luggen M. Cognitive dysfunction in SLE: development of a screening tool. Lupus. 2011;20(11):1142-6. doi:10.1177/0961203311405374

18. Kozora E, Arciniegas D, Zhang L, West S. Neuropsychological patterns in systemic lupus erythematosus patients with depression. Arthritis Res Ther. 2007;9(3):R48. doi:10.1186/ar2203

19. Kozora E, Hanly J, Lapteva L, Filley C. Cognitive dysfunction in systemic lupus erythematosus: past, present, and future. Arthritis Rheum. 2008;58(11):3286-98. doi:10.1002/art.23991

20. Peretti CS, Peretti CR, Kozora E, Papathanassiou D, Chouinard VA, Chouinard G. Cognitive impairment in systemic lupus erythematosus women with elevated autoantibodies and normal single photon emission computerized tomography. Psychother Psychosom. 2012;81(5):276-85. doi:10.1159/000336555

21. Faust T, Chang E, Kowal C, Berlin R, Gazaryan IG, Bertini E et al. Neurotoxic lupus autoantibodies alter brain function through two distinct mechanisms. Proc Natl Acad Sci USA. 2010;107(43):18569-74. doi:10.1073/pnas.1006980107

22. Lauvsnes MB, Beyer MK, Kvaloy JT, Greve OJ, Appenzeller S, Kvivik I et al. Hippocampal atrophy is associated with cerebrospinal fluid anti-NR2 antibodies in patients with systemic lupus erythematosus and primary Sjögren's syndrome. Arthritis Rheum. 2014;66(12):3387-94. doi:10.1002/art.38852

23. Petri M, Naqibuddin M, Sampedro M, Omdal R, Carson KA. Memantine in systemic lupus erythematosus: a randomized, double-blind placebo-controlled trial. Semin Arthritis Rheum. 2011;41(2):194-202. doi:10.1016/j.semarthrit.2011.02.005

24. Appenzeller S, Cendes F, Costallat LTL. Epileptic seizures in systemic lupus erythematosus. Neurology. 2004;63(10):1808-12. doi:10.1212/01.WNL.0000144178.32208.4F

25. Nabbout R. Autoimmune and inflammatory epilepsies. Epilepsia. 2012;53 Suppl 4:58-62. doi:10.1111/j.1528-1167.2012.03614.x

26. Mikdashi J, Krumholz A, Handwerger B. Factors at diagnosis predict subsequent occurrence of seizures in systemic lupus erythematosus. Neurology. 2005;64(12):2102-7. doi:10.1212/01.WNL.0000165959.98370.D5

27. Merayo-Chalico J,Apodaca E, Barrera-Vargas A, Alcocer-Varela A, Colunga-Pedraza I, González-Patiño A et al. Clinical outcomes and risk factors for posterior reversible encephalopathy syndrome in systemic lupus erythematosus: a multicentric case-control study.J Neurol Neurosurg Psychiatry. 2015;87(3):587-94. doi:10.1136/jnnp-2014-310145

28. Hirohata S, Arinuma Y, Yanagida T, Yoshio T. Blood-brain barrier damages and intrathecal synthesis of anti-N-methyl-D-aspartate receptor NR2 antibodies in diffuse psychiatric/neuropsychological syndromes in systemic lupus erythematosus. Arthritis Res Ther. Arthritis Res Ther. 2014;16(2):R77. doi:10.1186/ar4518

29. Nishimura K, Omori M, Horikawa N, Tanaka E, Furuya T, Harigai M. Risperidone in the treatment of acute confusional state (delirium) due to neuropsychiatric lupus erythematosus: case report. Int J Psychiatry Med]. 2003;33(3):299-303. doi:10.2190/P2T8-Y12E-ANKM-8MWF

30. Palagini L, Mosca M, Tani C, Gemignani A, Mauri M, Bombardieri S. Depression and systemic lupus erythematosus: a systematic review. Lupus. 2013;22(5):409-16. doi:10.1177/0961203313477227

31. Postal M, Lapa AT, Sinicato NA, Peliçari KO, Peres FA, Costallat LT, et al. Depressive symptoms are associated with tumor necrosis factor alpha in systemic lupus erythematosus. J Neuroinflammation. 2016;13(5):5. doi:10.1186/s12974-015-0471-9
32. Kivity S, Agmon-Levin N, Zandman-Goddard G, Chapman J, Shoenfeld Y. Neuropsychiatric lupus: a mosaic of clinical presentations. BMC Med. 2015;13(1):43. doi:10.1186/s12916-015-0269-8

33. Holmqvist M, Simard JF, Asplund K, Arkema EV. Stroke in systemic lupus erythematosus: a meta-analysis of population-based cohort studies. RMD Open. 2015;1:e000168. doi:10.1136/rmdopen-2015-000168

34. Mikdashi J, Handwerger B, Langenberg P, Miller M, Kittner S. Baseline disease activity, hyperlipidemia, and hypertension are predictive factors for ischemic stroke and stroke severity in systemic lupus erythematosus. Stroke. 2007;38(2):281-5. doi:10.1161/01.STR.0000254476.05620.14

35. Panichpisal K, Rozner E, Levine SR. The management of stroke in antiphospholipid syndrome. Curr Rheumatol Rep. 2012;14(1):99-106. doi:10.1007/s11926-011-0223-5

36. Sciascia S, Sanna G, Khamashta MA, Cuadrado MJ, Erkan D, Andreoli L et al. The estimated frequency of antiphospholipid antibodies in young adults with cerebrovascular events: a systematic review. Ann Rheum Dis. 2015;74(11):2028-33. doi:10.1136/annrheumdis-2014-205663

37. Urbanus RT, Siegerink B, Roest M, Rosendaal FR, Groot PG, Algra A. Antiphospholipid antibodies and risk of myocardial infarction and ischaemic stroke in young women in the RATIO study: a case-control study. Lancet Neurol. 2009;8(11):998-1005. doi:10.1016/S1474-4422(09)70239-X

38. Behrouz R. The risk of ischemic stroke in major rheumatic disorders. J Neuroimmunol. 2014;277(1-2):1-5. doi:10.1016/j.jneuroim.2014.09.009

39. Baizabal-Carvallo JF, Bonnet C, Jankovic J. Movement disorders in systemic lupus erythematosus and the antiphospholipid syndrome.J Neural Transm. 2013;120(11):1579-89. doi:10.1007/s00702-013-1023-z

40. Cardoso F. Movement disorders in childhood. Parkinsonism Relat Disord. 2014;20 Suppl 1:S13-16. doi:10.1016/S1353-8020(13)70006-3

41. Barsottini OG, Ferraz HB, Seviliano MM, Barbieri A. Brain SPECT imaging in Sydenham's chorea. Braz J Med Biol Res. 2002;35(4):431-6. doi:10.1590/S0100-879×2002000400004

42. Martino D, Chew NK, Mir P, Edwards MJ, Quinn NP, Bhatia KP. Atypical movement disorders in antiphospholipid syndrome. Mov Disord. 2006;21(7):944-9. doi:10.1002/mds.20842

43. Baizabal-Carvallo J, Delgadillo-Márquez G, Estañol B, Garcia-Ramos G. Clinical characteristics and outcomes of the meningitides in systemic lupus erythematosus. Eur Neurol. 2009;61(3):143-8. doi:10.1159/000186504

44. Schulz SW, Shenin M, Mehta A, Kebede A, Fluerant M, Derk CT. Initial presentation of acute transverse myelitis in systemic lupus erythematosus: demographics, diagnosis, management and comparison to idiopathic cases. Rheumatol Int. 2012;32:2623-7. doi:10.1007/s00296-011-2053-1

45. Fangtham M, Petri M. 2013 update: hopkins lupus cohort. Curr Rheumatol Rep. 2013;15(9):360. doi:10.1007/s11926-013-0360-0

46. Saison J, Costedoat-Chalumeau N, Maucort-Boulch D, Iwaz J, Marignier R, Cacoub P et al. Systemic lupus erythematosus-associated acute transverse myelitis: manifestations, treatments, outcomes, and prognostic factors in 20 patients. Lupus. 2015;24(1):74-81. doi:10.1177/0961203314547795

47. Lacomis D, Zivković SA. Approach to vasculitic neuropathies. J Clin Neuromuscul Dis. 2007;9(1):265-76. doi:10.1097/CND.0b013e31815202b3

48. Moore PM, Fauci AS. Neurologic manifestations of systemic vasculitis. A retrospective and prospective study of the clinicopathologic features and responses to therapy in 25 patients. Am J Med. 1981;71(4):517-24. doi:10.1016/0002-9343(81)90194-7 
49. Oomatia A, Fang H, Petri M, Birnbaum J. Peripheral neuropathies in systemic lupus erythematosus: clinical features, disease associations, and immunologic characteristics evaluated over a twenty-five-year study period. Arthritis Rheumatol. 2014;66(4):1000-9. doi:10.1002/art.38302

50. Khan S, Zhou L. Characterization of non-length-dependent small-fiber sensory neuropathy. Muscle Nerve. 2012;45(1):86-91. doi:10.1002/mus.22255

51. Servioli L, Pérez C, Consani S, Suárez A, Sehabiaga G, Collazo C et al. Prevalence and characteristics of immunomediated neuropathies in a group of patients with autoimmune diseases. J Clin Neuromuscul Dis. 2007;9(2):285-90. doi:10.1097/CND.0b013e318157614b

52. Florica B, Aghdassi E, Su J, Gladman DD, Urowitz MB, Fortin PR. Peripheral neuropathy in patients with systemic lupus erythematosus. Semin Arthritis Rheum. 2011;41(2):203-11. doi:10.1016/j.semarthrit.2011.04.001

53. Hantson P, Kevers L, Fabien N, Van Den Bergh P. Acute-onset chronic inflammatory demyelinating polyneuropathy with cranial nerve involvement, dysautonomia, respiratory failure, and autoantibodies. Muscle Nerve. 2010;41(3):423-6. doi:10.1002/mus.21543

54. Hsu TY, Wang SH, Kuo CF, Chiu TF, Chang YC. Acute inflammatory demyelinating polyneuropathy as the initial presentation of lupus. Am J Emerg Med. 2009;27(7):900.e3-5. doi:10.1016/j.ajem.2008.11.006

55. Gaber W, Ezzat Y, El Fayoumy NM, Helmy H, Mohey AM. Detection of asymptomatic cranial neuropathies in patients with systemic lupus erythematosus and their relation to antiribosomal $P$ antibody levels and disease activity. Clin Rheumatol. 2014;33(10):1459-66. doi:10.1007/s10067-014-2679-y

56. Roverano S, Cassano G, Paira S, Chiavarini J, Graf C, Rico L et al. Asymptomatic sensorineural hearing loss in patients with systemic lupus erythematosus. J Clin Rheumatol. 2006;12(5):217-20. doi:10.1097/01.rhu.0000242777.71604.6

57. Magro Checa C, Cohen D, Bollen EL, Buchem MA, Huizinga TW, Steup-Beekman GM. Demyelinating disease in SLE: is it multiple sclerosis or lupus? Best Pract Res Clin Rheumatol. 2013;27(3):405-24. doi:10.1016/j.berh.2013.07.010

58. Lin YC, Wang AG, Yen MY. Systemic lupus erythematosus-associated optic neuritis: clinical experience and literature review. Acta Ophthalmol. 2009;87(2):204-10. doi:10.1111/j.1755-3768.2008.01193.x

59. Karatas E, Onat AM, Durucu C, Baglam T, Kanlikama M, Altunoren O et al. Audiovestibular disturbance in patients with systemic lupus erythematosus. Otolaryngol Head Neck Surg. 2007;136(1):82-6. doi:10.1016/j.otohns.2006.06.1255

60. Batuecas-Caletrío A, Pino-Montes J, Cordero-Civantos C, Calle-Cabanillas MI, Lopez-Escamez JA. Hearing and vestibular disorders in patients with systemic lupus erythematosus. Lupus. 2013;22(5):437-42. doi:10.1177/0961203313477223

61. Zirkzee EJ, Steup-Beekman GM, Mast RC, Bollen EL, Wee NJ, Baptist E et al. Prospective study of clinical phenotypes in neuropsychiatric systemic lupus erythematosus; multidisciplinary approach to diagnosis and therapy. J Rheumatol. 2012;39(11):2118-26. doi:10.3899/jrheum.120545 